\title{
PROCESO DE ATENCIÓN PSICOLÓGICA EN EL PROGRAMA DE ATEN- CIÓN Y CUIDADOS EN ONCOLOGÍA DEL INSTITUTO NACIONAL DE CANCEROLOGÍA
}

\author{
THE PSYCHOLOGICAL PROCESS IN PROGRAM OF CARE IN ONCOLOGY IN THE \\ NATIONAL CANCER INSTITUTE
}

Leticia Ascencio-Huertas, Nancy Elizabeth Rangel-Domínguez y Silvia Allende Pérez

Servicio de Cuidados Paliativos. Instituto Nacional de Cancerología, México

Resumen

Enfrentarse a un cáncer avanzado implica un gran malestar físico y emocional para el paciente y su familia, es por ello que la atención psicológica es una prioridad en los cuidados paliativos. La terapia cognitivo-conductual es uno de los marcos teóricos más aplicados en la atención del paciente paliativo y tiene por objetivo la modificación de pensamientos y conductas respecto a la enfermedad que resultan disfuncionales y que impactan negativamente en el bienestar del paciente y sus familiares. El objetivo del estudio fue describir el proceso de atención psicológica en el Programa de Atención y Cuidados en Oncología del Instituto Nacional de Cancerología, así como las caracteristicas generales, diagnósticos oncológicos y psicológicos; y las técnicas de intervención psicológica más empleadas. Se realizo un estudio retrospectivo, trasnversal y descriptivo de la población atendida de febrero del 2011 a marzo del 2013. Con una muestra total de $\mathbf{5 . 5 8 8}$ pacientes. Se procesaron los datos a traves del análisis de frecuencias, obteniendo que dentro de los diagnósticos oncológicos más frecuentes el cáncer de estómago, mama y cervicouterino; encontrando en la mayoría de ellos, impacto emocional y procesos adaptativos al encontrarse fuera de tratamiento oncológico y cuyas técnicas psicológicas más aplicadas fueron: a) Psicoeducación, b) Expresión y regulación

\section{Abstract}

Coping in advanced cancer involves physical problems and emotional discomfort for the patient and his family, which is why psychological care care is a priority in palliative care. Cognitive behavioral therapy is one of the theoretical frameworks applied in the care of palliative patients and aims modifying thoughts and behaviors regarding the disease that are dysfunctional and negative impact the welfare of the patient and family. The aim of the study was to describe the psychological process in Program of Care in Oncology in The National Cancer Institute, general characteristic, cancer and psychological diagnoses and psychological techniques most used. We performed a retrospective study trasnversal and descriptive in population attended from February 2011 to March 2013. Total sample was 5588 patients. Data were processed with frequency analysis. The types of cancer most frequently attended were: gastrointestinal, breast and cervical, finding most of them with impact and adaptive processes are outside the oncology treatment. The most widely used psychological techniques were:a) Psychoeducation, b) Emotional expression and regulation c) emotional validation, d) Emotional containment, and e) Problem-solving.

Key words: Psychological intervention, cognitive-behavioral, palliative care.

\section{Correspondencia:}

Leticia Ascencio Huertas.

Av. San Fernando 22 Belisario Domínguez Sección XVI, Tlalpan, 14080. Ciudad de México, Distrito Federal. México.

E-mail: leash71@yahoo.com.mx 


\section{emocional c) Validación emocional, d) Conten- ción emocional, y e) Solución de problemas.}

Palabras clave: Intervención psicológica, cognitivo-conductual, cuidados paliativos.

\section{INTRODUCCIÓN}

En México durante el 2008, 77.700 miles de mexicanos de ambos sexos murieron por cáncer ${ }^{(1)}$ lo cual supone que los pacientes se enfrentaron en algún momento a un padecimiento progresivo e incurable, sin respuesta a tratamiento curativo y con múltiples síntomas. Muchos de estos pacientes pudieron beneficiarse de la atención proporcionada por los cuidados paliativos, ya que al no existir posibilidades de curación, la paliación es la alternativa de atención más viable ${ }^{(2)}$.

Las guías de The National Comprehensive Cancer Network definen Cuidados paliativos como el apoyo que se brinda al paciente con una enfermedad grave o potencialmente mortal para mejorar la calidad de vida a través de la prevención temprana y el tratamiento de los síntomas y los efectos secundarios causados por una enfermedad y su tratamiento ${ }^{(3)}$.

La atención psicológica es una prioridad en los servicios de cuidados paliativos para incrementar el bienestar del paciente, incluyendo los temas de autoestima, visión y adaptación a la enfermedad y sus consecuencias, la comunicación, la expresión emocional y psicológica, así como el funcionamiento social, tomando en cuenta la cultura, los valores, las creencias, la esfera espiritual, los factores sociales del paciente, y también los aspectos prácticos, como las finanzas y la vivienda ${ }^{(4,5)}$.

Enfrentarse a un cáncer avanzado implica un gran malestar físico y emocional para el paciente y su familia. Las personas con cáncer avanzado presentan con mayor frecuencia depresión, trastornos de ansie- dad y altos niveles de distrés en comparación con la población en general ${ }^{(6-12)}$ por lo que las intervenciones psicológicas son indispensables en los cuidados paliativos ${ }^{(13)}$.

Por otra parte, la familia del paciente con cáncer, y el cuidador primario, se enfrentan a constantes retos y responsabilidades que impactan sobre su propia salud y calidad de vida, presentan fatiga, problemas de sueño, depresión, ansiedad, agotamiento y mayor riesgo de mortalidad ${ }^{(14-19)}$. Las familias de pacientes en cuidados paliativos se enfrentan además a problemas sociales, familiares y financieros al tiempo que hacen frente a la inminente muerte de su ser querido ${ }^{(20-23)}$.

Ante las necesidades psicológicas de los pacientes con cáncer avanzado, la labor del especialista de la Psicología en cuidados paliativos cobra una relevante importancia, ya que dentro de sus principales objetivos está promover la mejora de la calidad de vida en el paciente y su familia, ayudar en la identificación y control de síntomas psicológicos y fomentar el uso de estrategias de afrontamiento efectivas respecto a la enfermedad y la inminente posibilidad de muerte ${ }^{(24)}$.

Sin embrago no solo la atención emocional del pacientes y su familia son parte de las actividades del psicólogo especialista en cuidados paliativos, sino también la asistencia a los miembros del equipo de salud, la docencia y la investigación. Algunos estudios hacen referencia a estas actividades. Uno de ellos es el de Jünger et al. ${ }^{(25)} \mathrm{y}$ el de Romero et al. ${ }^{(26)}$ en los que se afirma que la mayor parte de las actividades asistenciales consistían en la evaluación y manejo de trastornos depresivos, 
ansiosos y adaptativos presentados por el paciente y la familia, así como de identificar y atender las necesidades y creencias respecto a la enfermedad, la alta demanda de cuidados, el deterioro físico y la incapacidad para alimentarse o realizar actividades cotidianas y la muerte.

Algunos autores especializados en el campo, como Rainbird et al. ${ }^{(27)}$ indican que algunas de las principales necesidades del paciente con cáncer avanzado son primordialmente de tipo emocional, de información y comunicación, por tanto las intervenciones deben estar basadas en la empatía y la escucha activa, en la información sobre lo que espera con respecto a la enfermedad y en brindar la condiciones para poder expresar sus emociones al respecto; ya que se ha comprobado que estas acciones promueven el bienestar psicológico ${ }^{(28)}$.

Una de las consideraciones importantes con lo que respecta a las intervenciones psicológicas en Cuidados Paliativos es que debe ser realizadas por psicólogos clínicos entrenados en el campo de la salud, cuyo marco teórico permita realizar intervenciones breves, fáciles de aprender y diseñadas de manera particular a cada paciente $y$ su familia; tomando en consideración la presencia de múltiples síntomas como el dolor, problemas de concentración, fatiga, debilidad, anorexia y problemas de sueño, entre otros que pueden repercutir en el proceso terapéutico ${ }^{(29)}$.

Con respecto a la familia, Barreto ${ }^{(30)}$ reporta que algunos de los objetivos de atención psicológica están orientados a brindar soporte emocional ante la necesidad de información con respecto a la enfermedad y su evolución, el apoyo instrumental y emocional al paciente en casa, y la comunicación entre los integrantes de la familia, el paciente y el equipo de salud; identificar y atender el impacto que la enfermedad ejerce sobre otros miembros de la familia y proporcionar apoyo psicológico ante la cercanía de la pérdida de un ser querido; estas tareas se basan en el reconocimiento y fortalecimiento de las capacidades y necesidades de la familia respecto a su participación en los cuidados del paciente, al tiempo que se promueve una relación de confianza y colaboración entre la familia y el equipo de salud(31).

Dentro de los marcos teóricos que más se han aplicada en la atención del paciente oncológico en las diferentes etapas y en los escenarios paliativos se encuentra la terapia cognitivo-conductual, que combina una serie de estrategias de corte cognitivo (como la identificación y modificación de pensamientos disfuncionales respecto a la enfermedad y sus tratamientos) y estrategias de corte conductual (como el diseño y ejecución de un plan de activación diario y la distracción conductual) que inciden de manera directiva y rápida en la atención de las necesidades del paciente y su familia(32).

Dentro de la técnicas cognitivo conductuales más utilizadas en las intervenciones psicológicas proporcionadas a los pacientes oncológicos en fase avanzada y su familia se encuentran:

La psicoeducación, que consiste en una estrategia de enseñanza-aprendizaje para la salud que se basa en el aprendizaje experiencial sobre la enfermedad que se padece y las formas en que se pueden afrontar las consecuencias de tal padecimiento. Los objetivos de la psicoeducación son: 1) que el paciente participe de manera activa en el proceso de tratamiento y en sus propios cuidados, 2) generar conciencia sobre la enfermedad y la importancia del tratamiento y 3) favorecer la reducción de malestar emocional ante procedimientos. La psicoeducación ha mostrado ser efectiva en el manejo del dolor como tratamiento adyuvante a los medicamentos, controlar el distrés asociado a la enfermedad e incrementar el bienestar y mejorar la calidad de vida ${ }^{(33-35)}$. 
Con respecto a la familia se ha demostrado que el proveer de información con respecto a los síntomas más frecuentes, el control de los mismos y la atención emocional del paciente impacta positivamente en la confianza y habilidades de atención de los familiares hacia sus pacientes ${ }^{(36)}$.

Otra de las técnicas más utilizadas es la expresión y regulación emocional. La expresión de emociones puede presentarse como un problema fundamental en la atención del paciente oncológico y con enfermedad terminal, ya que se ha demostrado que el paciente con cáncer tienden a reprimir emociones, principalmente las consideradas negativas como el miedo y el enojo, lo que constituye una mayor dificultad en su identificación y por tanto en la atención que la familia pueda brindar a las necesidades emocionales del paciente ${ }^{(31,37-40)}$.

Sin embargo, es importante mencionar que, no solo es necesario que los pacientes y sus familiares expresen sus emociones sino también que lo hagan de manera adecuada, es decir que las regulen. Se entiende como regulación emocional la habilidad para modular el afecto (respecto a su inicio, intensidad y tiempo de presentación) siguiendo las normas sociales y culturales al respecto y que permite alcanzar metas personales ${ }^{(41)}$.

Por lo que la identificación y entrenamiento para la expresión efectiva de las emociones constituye un área de intervención del profesional de la Psicología, debido a que si son atendidas de manera efectiva repercuten en el bienestar percibido por el paciente y su familia ${ }^{(42)}$.

Dentro de la técnicas que requieren una atención específica se encuentran las técnicas de relajación cuyas indicaciones y contraindicaciones deben ser muy bien conocidas por el psicólogo especialista en cuidados paliativos debido a la presencia de síntomas físicos como la disnea y el dolor, que deben ser considerandos antes de proponer alguna de estas técnicas.
Estas intervenciones buscan que los pacientes y sus familiares experimenten sensaciones confortables e incompatibles con las respuestas de distrés emocional ${ }^{(43)}$ su efectividad se ha identificado en el manejo dolor crónico, al reducir la intensidad del mismo $^{(44)}$, en la reducción y duración de síntomas como la nausea y el vómito, así como un manejo adecuado de la ansiedad asociada a disnea ${ }^{(45)}$.

Otra de la técnicas cognitivas utilizadas en el contexto paliativo, es la terapia de solución de problemas, que se enfoca en ayudar a los pacientes y sus familiares desarrollar habilidades para la identificación de problemas y la elaboración de posibles soluciones ${ }^{(46)}$. Las intervenciones basadas en la solución de problemas frecuentemente están dirigidas a los cuidadores primarios ya que requiere condiciones cognitivas específicas, por esta razón su uso para algunos pacientes paliativos dependerá de sus condiciones físicas y mentales. Han sido empleadas en el manejo de síntomas y la búsqueda de información y han demostrado tener un impacto positivo sobre la calidad de vida de los cuidadores primarios $^{(47-49)}$.

Uno de los procesos de atención psicológica que suele ocuparse con mayor frecuencia en los escenarios oncológicos es la Psicoterapia de apoyo, que tiene por objetivo ayudar a los pacientes a hacer frente a las situaciones estresantes y las reacciones emocionales asociadas. Se basan en la identificación de las fortalezas previas de las personas y la promoción de estrategias de afrontamiento a la enfermedad. Estas intervenciones son altamente flexibles y se pueden modificar con cada paciente e incluso con el mismo paciente y familiar en situaciones diferentes ${ }^{(43)}$.

Dentro de este tipo de estrategias destaca la intervención en crisis, que se define como un proceso orientado a la resolución de uno o varios problemas derivados de una situación inesperada y que afecta 
negativamente el curso de la vida de una persona. Tiene por objetivos amortiguar el impacto de un evento estresante, ayudar a las personas a identificar y fortalecer las estrategias de afrontamiento, controlar $y$ disminuir el estado de estrés, ansiedad, desamparo y confusión del paciente, y finalmente, ayudarle a realizar actividades necesarias e inmediatas basadas en su condición física, social, familiar y contextual(50).

A lo largo del manejo paliativo pueden presentarse diversas y continuas situaciones de crisis, y no solo la confirmación de un diagnostico oncológico, la notificación de la imposibilidad de continuar el tratamiento curativo sino la pérdida de funciones, el deterioro físico, la pérdida del rol familiar y social, la pérdida de la autonomía y la dependencia hacia el otro (familia y/o equipo de salud). Ante lo cual es importante echar mano de todas la herramientas con las que se cuenta en área de atención psicológica para poder disminuir en el paciente el discomfort y cumplir con los objetivos primordiales de los cuidados paliativos: alcanzar una adecuada calidad de vida y morir con un satisfactorio control de síntomas, físicos, emocionales, sociales y espirituales,

Ante este panorama se propone el presente trabajo, que tiene por objetivo mostrar el proceso de atención y técnicas de intervención psicológica más utilizadas en el Servicio de Cuidados Paliativos del Instituto Nacional de Cancerología en una experiencia de 2 años de trabajo (de marzo del 2011 a febrero del 2013).

En el Instituto Nacional de Cancerología en México (INCan), el Servicio de Cuidados Paliativos existe desde hace más de dos décadas en conjunto con Clínica del Dolor, pero no es sino hasta el año 2010 que se formaliza la separación del Servicio de manera independiente y con ello se da pie a la creación del Programa de Atención y Cuidados en Oncología (PACO), en donde coadyuva el Área de Atención Psicológica; cuyo objetivo principal es la evaluación y atención de problemas emocionales de pacientes y familiares ante el avance de la enfermedad oncológica, desde la confirmación del diagnóstico, pasando por la evolución de la enfermedad, el encontrarse fuera de tratamiento oncológi$\mathrm{CO}$, el deterioro físico; hasta el fallecimiento y el manejo del duelo para los dolientes.

La ruta de atención por parte del Área de Psicología, está estrechamente relacionada con el Modelo Médico del Programa de Atención y Cuidados en Oncología establecido en el INCan; en donde se reciben pacientes en dos situaciones: 1) pacientes que acuden por primera vez al Instituto al servicio de Pre-consulta, en donde al ser valorados por un médico oncólogo se determina que se encuentran fuera de tratamiento oncológico y solo tienen la opción de manejo paliativo y 2) aquellos pacientes referidos por algunas de las áreas de especialización oncológica, en donde ya ha recibido tratamiento oncológico (quimioterapia, radioterapia o cirugía), pero que a pesar de ellos se encuentra ante progresión o recaída de la enfermedad oncológica sin posibilidad de continuar con alguno de los tratamientos.

Una vez que los pacientes ingresan al Programa PACO, son valorados por el Área Médica quien determina los síntomas a paliar y el tratamiento más adecuado ante las necesidades físicas del paciente. Posteriormente son referidos al Área de Psicología para determinar la condición mental y emocional del paciente y la familia. A través de una entrevista clínica inicial con el paciente y su familia se identifican las principales necesidades emocionales, la etapa del proceso de adaptación y/o alteraciones en el mismo, así como la capacidad para tomar decisiones. Se explica el objetivo de atención psicológica en cuidados paliativos, el manejo paciente/familia y se propone un tratamiento psicológico 
individualizado enfocado a sus necesidades y condiciones. Si el paciente acepta, la atención se lleva a cabo de forma presencial en la consulta externa o bien en sus ingresos hospitalarios, ya sea en hospitalización, hospital de día, o puede realizarse por vía telefónica y en visita domiciliaria. Es importante destacar que, debido a las necesidades de naturaleza cambiante de las condiciones del paciente, la intervención es dinámica y flexible, y se busca emplear las estrategias más adecuadas al momento.

\section{MÉTODO}

Se trata de una investigación retrospectiva, transversal y descriptiva. La información fue obtenida de la base de registro de actividades diarias del área de psicología. Se obtuvieron datos sociodemográficos (edad, genero, lugar de residencia), datos relacionados con la enfermedad oncológica (diagnóstico oncológico y servicio médico de base que refiere al paciente al Programa PACO del
Servicio de Cuidados Paliativos) y datos del proceso de atención e intervención psicológica (área de atención, diagnóstico psicológico y técnicas psicológicas utilizadas). Se reportan los datos recabados durante un período de 2 años: de febrero del 2011 a marzo del 2013. Los datos fueron sometidos a un análisis de frecuencias a través del paquete estadístico SPSS versión 19.0.

\section{RESULTADOS}

La población estudiada fue de 5588 pacientes, en su mayoría mujeres, principalmente casados (ver tabla 1) Con respecto a la edad se encontró que la población estudiada se encuentra entre la quinta y la sexta década de la vida, con una edad promedio cercana a los 59 años (ver tabla 2) Los pacientes atendidos eran residentes del Distrito Federal y algunos estados cercanos, como el Estado de México e Hidalgo (ver tabla 3) sin embargo se atendieron pacientes originarios de casi todas la regiones de México.

\section{Tabla 1. Características de la población}

\begin{tabular}{|l|c|c|}
\hline & Frecuencia & Porcentaje \\
\hline \multicolumn{3}{|c|}{ Género } \\
\hline Femenino & 3.302 & 59,1 \\
\hline Masculino & 2.286 & 40,9 \\
\hline \multicolumn{3}{|c|}{ Estado Civil } \\
\hline Solter@ & $\mathbf{1 . 0 3 0}$ & $\mathbf{1 8 , 4}$ \\
\hline Casad@ & $\mathbf{2 . 6 7 2}$ & $\mathbf{4 7 , 8}$ \\
\hline Divorciad@ & 147 & 2,6 \\
\hline Unión Libre & 424 & 7,6 \\
\hline Viud@ & $\mathbf{8 2 6}$ & $\mathbf{1 4 , 8}$ \\
\hline Separad@ & 489 & 8,8 \\
\hline \multicolumn{3}{|c|}{ Primera Vez / Subsecuente } \\
\hline Primera Vez & 2.193 & 39,2 \\
\hline Subsecuente & $\mathbf{3 . 3 9 5}$ & $\mathbf{6 0 , 8}$ \\
\hline Total & 5.588 & 100,0 \\
\hline
\end{tabular}




\section{Tabla 2. Edad (Frecuencias y Medidas de Tendencia Central)}

\begin{tabular}{|l|c|c|}
\hline Rango en años & Frecuencia & Porcentaje \\
\hline 16 a 20 & 97 & 1,7 \\
\hline 21 a 30 & 251 & 4,5 \\
\hline 31 a 40 & 479 & 8,6 \\
\hline 41 a 50 & 939 & 16,8 \\
\hline 51 a 60 & $\mathbf{1 . 2 2 5}$ & 21,9 \\
\hline 61 a 70 & 1.218 & 21,8 \\
\hline 71 a 80 & 903 & 16,2 \\
\hline 81 a 90 & 447 & 8,0 \\
\hline 91 a 100 & 28 & 0,5 \\
\hline 100 a 105 & 1 & 0,0 \\
\hline Total & 5.588 & 100 \\
\hline
\end{tabular}

Mínimo 16, Máximo 105; Media 58,8; Desviación Típica 16,382

Tabla 3. Lugar de Residencia

\begin{tabular}{|l|c|c|}
\hline Estados & Frecuencia & Porcentaje \\
\hline Chiapas & 40 & 0,7 \\
\hline Distrito Federal & $\mathbf{2 . 3 5 8}$ & $\mathbf{4 2 , 2}$ \\
\hline Estado de México & $\mathbf{1 . 7 9 7}$ & $\mathbf{3 2 , 2}$ \\
\hline Guerrero & 90 & 1,6 \\
\hline Hidalgo & $\mathbf{4 5 5}$ & $\mathbf{8 , 1}$ \\
\hline Michoacán & 92 & 1,6 \\
\hline Morelos & 257 & 4,6 \\
\hline Oaxaca & 83 & 1,5 \\
\hline Puebla & 92 & 1,6 \\
\hline Querétaro & 55 & 1,0 \\
\hline Tlaxcala & 98 & 1,8 \\
\hline Veracruz & 87 & 1,6 \\
\hline
\end{tabular}

En la tabla 4 se observan los servicios oncológicos de base que refieren pacientes al Servicio de Cuidados Paliativos, entre los que destacan el servicio de Oncología Médica, la Preconsulta, Gastroenterología y Ginecología. Estos datos concuerdan con los diagnósticos oncológicos registrados con mayor frecuencia, ya que destacan el cáncer gástrico y de esófago, así como los sarcomas de mama y el cáncer cervicouterino (ver tabla 5)
Las principales áreas de atención psicológica fueron: la consulta externa, la asistencia vía telefónica y la hospitalización (ver tabla 6) Los diagnósticos (basados en el DSM-IV y los manejos por el área de atención psicológica en Cuidados Paliativos) registrados con mayor frecuencia fueron el proceso de adaptación al tratamiento paliativo, seguido por el diagnóstico de duelo normal e impacto emocional ante diagnóstico y pronóstico (ver tabla 7) 
Tabla 4. Servicio oncológico que refiere al Servicio de Cuidados Paliativos

\begin{tabular}{|l|c|c|}
\hline & Frecuencia & Porcentaje \\
\hline Ginecología & $\mathbf{3 0 3}$ & $\mathbf{5 , 4}$ \\
\hline Piel y Partes Blandas & 129 & 2,3 \\
\hline Mama & 139 & 2,5 \\
\hline Cabeza y cuello & 124 & 2,2 \\
\hline Hematología & 214 & 3,8 \\
\hline Gastroenterología & $\mathbf{5 3 2}$ & $\mathbf{9 , 5}$ \\
\hline Neumología & 97 & 1,7 \\
\hline Oncología Médica & $\mathbf{2 . 2 3 8}$ & $\mathbf{4 0 , 1}$ \\
\hline Urología & 236 & 4,2 \\
\hline Preconsulta & $\mathbf{1 . 5 7 6}$ & $\mathbf{2 8 , 2}$ \\
\hline Total & 5.588 & 100,0 \\
\hline
\end{tabular}

\section{Tabla 5. Tipo de cáncer más frecuente en la población atendida}

\begin{tabular}{|l|c|c|}
\hline & Frecuencia & Porcentaje \\
\hline $\begin{array}{l}\text { Cáncer cavidad bucal, laringe, faringe y } \\
\text { glándulas salivales }\end{array}$ & 108 & 1,9 \\
\hline Cáncer de pulmón & 239 & 4,3 \\
\hline Cáncer de esófago y gástrico & $\mathbf{7 4 5}$ & $\mathbf{1 3 , 3}$ \\
\hline Cáncer intestino delgado y colorectal & 382 & 6,8 \\
\hline Cáncer de hígado & 297 & 5,3 \\
\hline Cáncer de vesícula y vías biliares & 161 & 2,9 \\
\hline Cáncer de páncreas & 390 & 7,0 \\
\hline $\begin{array}{l}\text { Sarcomas de mama, in situ y cáncer de } \\
\text { mama }\end{array}$ & $\mathbf{5 4 2}$ & $\mathbf{9 , 7}$ \\
\hline Cáncer cérvico-uterino & $\mathbf{5 0 5}$ & $\mathbf{9 , 0}$ \\
\hline Carcinoma de endometrio & 58 & 1,0 \\
\hline Cáncer de ovario & 265 & 4,7 \\
\hline Cáncer de riñón & 285 & 5,1 \\
\hline Cáncer de próstata & 182 & 3,3 \\
\hline Carcinomas de la piel y melanomas & 160 & 2,9 \\
\hline Sarcomas de tejidos blandos & 103 & 1,8 \\
\hline Linfomas & 227 & 4,1 \\
\hline Primario Desconocido & 115 & 2,1 \\
\hline Otros & 447 & 8,0 \\
\hline
\end{tabular}

Tabla 6. Área de atención psicológica

\begin{tabular}{|l|c|c|}
\hline & Frecuencia & Porcentaje \\
\hline Hospitalización & $\mathbf{9 7 1}$ & $\mathbf{1 7 , 4}$ \\
\hline Consulta externa & $\mathbf{2 . 0 4 1}$ & $\mathbf{3 6 , 5}$ \\
\hline Asistencia telefónica & $\mathbf{1 . 1 8 8}$ & $\mathbf{2 1 , 3}$ \\
\hline Visita Domiciliaria & 196 & 3.5 \\
\hline Unidad de Terapia Intensiva & 7 & 0,1 \\
\hline Atención inmediata & 119 & 2,1 \\
\hline Sesión Psicoeducativa & 307 & 5,5 \\
\hline Sesión familiar & 759 & 13.6 \\
\hline Total & 5.588 & 100 \\
\hline
\end{tabular}




\section{Tabla 7. Diagnósticos psicológicos basados en el DSM-IV-TR y condiciones de atención psicológica en cuidados paliativos}

\begin{tabular}{|l|c|c|}
\hline & Frecuencia & Porcentaje \\
\hline Impacto ante el diagnóstico y pronóstico & $\mathbf{7 3 3}$ & $\mathbf{1 3 , 1}$ \\
\hline Proceso de adaptación al tratamiento y pronóstico & $\mathbf{2 . 0 1 1}$ & $\mathbf{3 6 , 0}$ \\
\hline Impacto emocional ante progresión o recaída & 441 & 7,9 \\
\hline $\begin{array}{l}\text { Impacto emocional por estar fuera de tratamiento } \\
\text { oncológico }\end{array}$ & 470 & 8,4 \\
\hline Duelo anticipado complicado & 74 & 1,3 \\
\hline Duelo anticipatorio & 550 & 9,8 \\
\hline Reacciones a estrés grave y trastornos de adaptación & 93 & 1,7 \\
\hline Patrón de comportamiento Tipo C & 13 & 0,2 \\
\hline Episodios Depresivos & 66 & 1,2 \\
\hline Trastorno Depresivo Recurrente & 20 & 0,4 \\
\hline Claudicación del Cuidador Primario & 95 & 1,7 \\
\hline Claudicación familiar & 37 & 0,7 \\
\hline Conspiración del Silencio & 53 & 0,9 \\
\hline Duelo Normal & $\mathbf{7 6 6}$ & $\mathbf{1 3 , 7}$ \\
\hline Duelo patológico & 75 & 1,3 \\
\hline
\end{tabular}

\section{Tabla 8. Tipo de abordaje psicológico}

\begin{tabular}{|l|c|c|}
\hline & Frecuencia & Porcentaje \\
\hline Entrevista inicial & $\mathbf{1 . 7 5 0}$ & $\mathbf{3 1 , 3}$ \\
\hline Historia clínica & 183 & 3,3 \\
\hline Evaluación psicométrica & 7 & 0,1 \\
\hline Abordaje psicoterapéutico & 336 & 6,0 \\
\hline Psicoterapia de apoyo & $\mathbf{1 . 7 7 9}$ & $\mathbf{3 1 , 8}$ \\
\hline Psicoterapia breve & 164 & 2,9 \\
\hline Psicoterapia familiar & 119 & 2,1 \\
\hline Taller de duelo & $\mathbf{3 6 3}$ & $\mathbf{6 , 5}$ \\
\hline Intervención en crisis & 143 & 2,6 \\
\hline Sesiones psicoeducativas & 347 & 6,2 \\
\hline Valoración del estado emocional & 171 & 3,1 \\
\hline Valoración familiar & 190 & 3,4 \\
\hline Consejería familiar & 36 & 0,6 \\
\hline Total & 5.588 & 100 \\
\hline
\end{tabular}

La psicoterapia de apoyo y la entrevista inicial fueron los abordajes psicológicos empleados más frecuentemente. Con respecto al abordaje más frecuente fue la psicoterapia de apoyo y la entrevista inicial (ver tabla 8). Las técnicas de intervención más empleadas fueron la psicoeducación, la expresión emocional, la validación, la contención emocional y la solución de problemas. La tabla 9 y la figura 1 presen- tan una descripción detallada de las intervenciones empleadas.

Las técnicas complementarias son aquellas que se combinan con las técnicas iniciales y que fueron registradas a partir de marzo del 2012; ya que nos percatamos que era necesario registrar las técnicas que mejoraban la intervención psicológica y no considerar una sola técnica; dentro de las más frecuentes se encuentran: la expresión 
Tabla 9. Técnica Psicológica inicial y/o única más frecuente

\begin{tabular}{|l|c|c|}
\hline & Frecuencia & Porcentaje \\
\hline Reestructuración cognitiva & 86 & 1,5 \\
\hline Distracción & 6 & 0,1 \\
\hline Psicoeducación & $\mathbf{1 . 5 5 4}$ & $\mathbf{2 7 , 8}$ \\
\hline Balance decisional & 79 & 1,4 \\
\hline Solución de problemas & $\mathbf{3 1 1}$ & $\mathbf{5 , 6}$ \\
\hline Validación emocional & $\mathbf{1 . 2 5 5}$ & $\mathbf{2 2 , 5}$ \\
\hline Expresión de emociones & $\mathbf{1 . 5 0 0}$ & $\mathbf{2 6 , 8}$ \\
\hline Relajación muscular progresiva & 16 & 0,3 \\
\hline Respiración diafragmática & 4 & 0,1 \\
\hline Imaginación guidada & 22 & 0,4 \\
\hline Inoculación de estrés & 2 & 0,0 \\
\hline Toma de decisiones & 43 & 0,8 \\
\hline Intervención en crisis & 27 & 0,5 \\
\hline Automonitoreo de emociones & 5 & 0,1 \\
\hline Registros conductuales & 10 & 0,2 \\
\hline Counselling & 89 & 1,6 \\
\hline Habilidades sociales & 2 & 0,0 \\
\hline Asertividad & 10 & 0,2 \\
\hline Modificación de conducta & 1 & 0,0 \\
\hline Contención emocional & $\mathbf{5 6 6}$ & $\mathbf{1 0 , 1}$ \\
\hline Total & 5.588 & 100 \\
\hline
\end{tabular}

Tabla 10. Frecuencia de las técnicas psicológicas complementarias

\begin{tabular}{|l|c|c|}
\hline Técnicas psicológicas & Frecuencia & Porcentaje \\
\hline Psicoeducación & $\mathbf{4 7 1}$ & $\mathbf{1 3 , 1}$ \\
\hline Balance decisional & 35 & 1,0 \\
\hline Solución de problemas & 135 & 3,8 \\
\hline Validación emocional & $\mathbf{1 . 1 9 3}$ & $\mathbf{3 3 , 8}$ \\
\hline Expresión de emociones & $\mathbf{1 6 9}$ & $\mathbf{4 , 8}$ \\
\hline Couselling & 71 & 1,7 \\
\hline Modificación de conducta & 14 & 0,3 \\
\hline Contención emocional & $\mathbf{2 3 4}$ & $\mathbf{6 , 6}$ \\
\hline
\end{tabular}

emocional, la psicoeducación y la contención emocional. Con lo podemos resaltar que en la intervención psicológica no solo se utiliza una técnica psicológica sino una combinación de más de 2 técnicas para poder brindar una mejor atención (ver tabla 2)

\section{CONCLUSIONES}

Dentro de las consideraciones finales al realizar este trabajo consideramos es importante resaltar la importancia de contar con el área de Psicología dentro del equi- 
po de cuidados paliativos debido a que son los especialistas que cuentan con las herramientas necesarias para la detección, diagnóstico y tratamiento de trastornos emocionales que presentan los pacientes oncológicos en fase avanzada o terminal y su familia. Por lo que debe ser psicólogos clínicos especializados en el campo de la salud y preferentemente en el campo oncológico para realizar un abordaje integral entre las herramientas y condiciones que cuenta el área psicológica asociada a la enfermedad médica de base.

Además de ser necesario crear una base de datos más completa que nos permita conocer la combinación de técnicas psicológicas que se utiliza con más frecuencia en cuidados paliativos. Así como poder hacer ampliar esta base de datos a otros centros de cuidados paliativos en el país, con el objetivo de contar con un mayor registro con respecto a la atención psicológica, para poder determinar de una manera más generalizada cuales son los marcos teóricos y los tipos de intervenciones y técnicas psicológicas que llevan a cabo en México.

Es importante también dentro de las intervenciones y técnicas psicológicas considerar el deterioro físico y la fatiga de los pacientes ya que pueden evitar que se inicien o continúen con un proceso de atención psicológica por lo cual se sugiere sean breves y dirigidas a las necesidades concretas del paciente. Además, de contemplar que todavía se tienen que romper con los mitos de que llevar un tratamiento psicológico está relacionado con una connotación social de "locura".

Para futuras investigaciones se sugiere contar con estrategias que nos permitan verificar la efectividad de las técnicas y ver como los propios pacientes pueden definir la modificación en su calidad de vida con la intervención psicológica, tal como lo describe la revisión sistemática realizada por Bradley et al. ${ }^{(51)}$ en la que muestran que se debe explorar en los pacientes cómo valoran el apoyo proporcionado en la forma de afrontar afrontar mejor la enfermedad terminal, el impacto en su calidad de vida y a la aceptación y manejo de las pérdidas y la muerte.

\section{REFERENCIAS BIBLIOGRÁFICAS}

1. International Agency for Research on Cancer. GLOBOCAN 2008. [Acceso el 25 de julio de 2013]. Disponible en: .http://globocan.iarc.fr/factsheets/populations/factsheet.asp? uno=484\#KEY

2. OMS (2002). Programas nacionales de lucha contra el cáncer: directrices sobre política y gestión. Ginebra, Organización Mundial de la Salud.

3. Levy $M H$, Back A, Benedetti C, Billings JA, Block S, Boston B et al. SM: NCCN clinical practice guidelines in oncology: Palliative care. J Natl Compr Canc Netw 2009;7:436-73

4. Halligan PW and Aylward M. The power of belief: Psychosocial influence on illness, disability and medicine. Oxford: Oxford University Press, 2006.

5. Lloyd-Williams M. Psychosocial issues in palliative care, 2nd ed. Oxford: Oxford University Press, 2008

6. Durkin I, Kearney M, O'Siorain L. Psychiatric disorder in a palliative care unit. Palliat Med 2003;17:212-8.

7. Greenberg L, Lantz MS, Likourezos A, Burack OR, Chichin E, Carter J. Screening for depression in nursing home palliative care patients. J Geriatr Psychiatry Neurol 2004; 17: 212-8

8. Wilson KG, Graham ID, Viola RA, Chater S, de Faye BJ, Weaver LA, et al. Structured interview assessment of symptoms and concerns in palliative care. Can J Psychiatry 2004;49:350-8.

9. Wilson KG, Chochinov HM, Skirko MG, et al. Depression and anxiety disorders in palliative cancer care. J Pain Symptom Manage 2007;33:118-29. 
10. Herbaut A, Reich M, Horner-Vallet D. Assessment of psychological distress in palliative care. About 50 observations. Infokara 2003;18:5-6.

11. Reeve J, Lloyd-Williams M, Dowrick C. Depression in terminal illness: The need for primary care-specific research. Fam Pract 2007;24:263-8.

12. Galfin JM, Watkins ER, Harlow T. Psychological distress and rumination in palliative care patients and their caregivers. J Palliat Med 2010;13:1345-8. Doi: 10.1177/0269216311414757

13. Bradley SE, Friezelle D, Johnson M. Patients' psychosocial experiences of attending specialist palliative day care: A systematic review. Palliat Med 2011; 25:210-28. Doi: 10.1177/0269216310389222.

14. Swore BA, Schumacher KL, Dodd M, Paul SM, Cooper B, Lee K, et al. Trajectories of fatigue in family caregivers of patients undergoing radiation therapy for prostate cancer. Res Nurs Health. 2009;32:2:12539. Doi: 10.1002/nur.20312 49

15. Wei-Chung EW, Tsai YY, Chang TW, Tsao C). Quality of sleep and quality of life in caregivers of breast cancer patient. Psychooncology 2007;16:950-5. Doi: 10.1002/pon.1167

16. Grov EK., Dahl A, Moum T, Fossa D. Anxiety, depression, and quality of life in caregivers of patients with cancer in late palliative phase. Ann Oncol 2005;16:1185-91. Doi: 10.1093/annonc/mdi210

17. Rivera HR. Depression symptoms in cancer caregivers. Clin J Oncol Nurs 2009; 13:195-202. Doi: 10.1188/09.CJON.195202

18. Carol M. Grandmothers who are caregivers are more prone to stress and depressive symptoms. New York: Medical Study News 2001;123-32.

19. Seira MP, Aller A, Calvo A. Morbilidad sentida y diagnosticada en cuidadores de pacientes inmovilizados de una zona de salud rural. Rev Esp Salud Pública 2002;76:713-21
20. Neugaard B, Andresen E, McKune S L, Jamoom E W. Health-related quality of life a National Sample of caregivers: Findings from the behavioral risk factor surveillance system. J Happiness Stud 2008;9:559-75. Doi: 10.1007/s10902008-9089-2

21. Nigenda G, López-Ortega M, Matarazzo C, Juárez-Ramírez C. La atención de los enfermos y discapacitados en el hogar. Retos para el sistema de salud mexicano. Salud Publica Mex 2007;49:286-94

22. Sherwood P R, Donovan H S, Given C W, Lu X, Given B A, Hricik A, et al. Predictors of employment and lost hours from work in cancer caregivers. Psychooncology 2008;17:6:598-605. Doi: 10.1002/ pon. 1287

23. Stefani D, Seidmann S, Pano C, Acrich L. Los cuidadores familiares de enfermos crónicos: sentimiento de soledad, aislamiento social y estilos de afrontamiento. Rev Latinoam Psicol 2003;35:55-65.

24. Barreto P. Cuidados Paliativos al enfermo de sida en situación terminal: enfoque multidisciplinar. Rev Psicol Gen Apl 1994;2:2001-8

25. Jünger $S$, Eggenberger $E$, Greenwood A, Payne S. Psychologists in palliative care in Europe: A discipline 'under construction'. Abstracts of the 6th Research Congress of the European Association for Palliative Care. Palliat Med 2010; 24:212-3.

26. Romero C, Álvarez M, Bayés R, Schröder M. ¿Cuándo se pide la intervención del psicólogo en una unidad de cuidados paliativos? Med Pal 2001;8:170-2

27. Rainbird K, Perkins J, Sanson-Fisher R, Rolfe I, Anseline P. The needs of patients with advanced, incurable cancer. $\mathrm{Br}$ J Cancer 2009;101:759-64. Doi: 10.1038/ sj.bjc. 6605235

28. Hudson P, Quinn P, O'Hanlon B, Aranda S. Family meetings in palliative care: Multidisciplinary clinical practice guidelines BMC Palliative Care 2008;7:12 Doi:10.1186/1472-684X-7-12 
29. Doyle D, Hanks G, Cherny N, Calman K, Cherny NI. The problem of suffering. In: Doyle D, Hanks G, Cherny N, Calman K., editors. Oxford textbook of palliative medicine, 3rd ed. Oxford: Oxford University Press, 2005, p.7-13.

30. Barreto MP, Bayés R. El psicólogo ante el enfermo en situación terminal. An Psicol 1990;6:169-80

31. Hudson PL, Remedios C, Thomas K. A systematic review of psychosocial interventions for carers of palliative care patients. BMC Palliat Care 2010;9:17. Doi: 10.1186/1472-684X-9-17

32. Mendez J, Maya A. Intervención cognitivo-conductual en cuidados paliativos pediátricos. Revista Mexicana de Algología 2009;6:20-4.

33. Gómez C. Psiquiatría clínica: diagnóstico y tratamiento en niños, adolescentes y adultos. $3^{\text {a }}$ ed. Bogotá: Editorial Médica Internacional, 2008.

34. Lugo I, Becerra AL, Reynoso L. Intervención psicológica para manejo de ansiedad ante la colocación de catéter puerto en mujeres con cáncer de mama. Psicooncología 2013;10: 177-184. Doi: 10.5209/ rev_PSIC.2013.v10.41956

35. Devine EC. Meta-analysis of the effect of psychoeducational interventions on pain in adults with cancer. Oncol Nurs Forum 2003;30:75-89. Doi: 10.1188/03. ONF.75-89

36. Schildmann E, Higginson I. Evaluating psycho-educational interventions for informal carers of patients receiving cancer care or palliative care: Strengths and limitations of different study designs Palliat Med 2011;345-56. Doi: 10.1177/0269216310389223.

37. Northouse L L, Katapodi M, Song L, Zhang L, Mood DW. Interventions with family caregivers of cancer patients. Meta-analysis of randomized trial. CA Cancer J Clin 2010;60;317-39. Doi: 10.3322/caac.20081.

38. Porro ML, Andrés ML, Rodríguez-Espíndola S. Regulación emocional y cáncer:
Utilización de la expresión y supresión emocional en pacientes oncológicos. Avances en Psicología latinoamericana 2012;30:341-55.

39. Bleiker EMA, Van Der Ploeg HM. Psychosocial factors in the etiology of breast cancer: Review of a popular link. Patient Educ Couns 1999;37:201-14.

40. Giese-Davis J, Spiegel D. Emotional expression and cancer progression. En: Davison K, Scherer R, Goldsmith $\mathrm{HH}$, editors. Handbook of affective sciences. Oxford: Oxford University Press, 2002.p.1053-82

41. Retana-Franco BE, Sánchez-Aragón R. Rastreando el pasado... formas de regular la felicidad, la tristeza, el amor, el enojo y el miedo. Univ. Psychol 2010;9:179-97

42. Cerezo MV, Ortíz-Tallo M, Cardenal V. Expresión de emociones y bienestar en un grupo de mujeres con cáncer de mama: una intervención psicológica. Rev Latinoam Psicol 2009;41:131-40

43. Tatsuo A. Psychotherapy for depression among patients with avanced cancer. Jpn J Clin 2012;42:1113-9. Doi:10.1093/jjco/ hys 152

44. Flores ME, González-Rojas IP, Troyo R, Valle MA Muñoz A, Vega G. Efectividad de las técnicas de relajación en la disminución del dolor crónico. Investigación en Salud 2004;6:75-81.

45. Hernández JC. Uso de la psicoterapia como manejo coadyuvante del dolor y otros síntomas asociados al cáncer. Rev Medica Sanitas 2012;15:8-18

46. Nezu A. Terapia de Solución de Problemas para pacientes con cáncer. Rev Mex Psicol 2001;18:185-92.

47. Bucher JA, Loscalzo M, Zabora J, Houts P, Hooker C, BrintzenhofeSzoc K. Problem-solving cancer care education for patients and caregivers. Cancer Pract 2001;9:66-70. Doi: 10.1046/j15235394.2001.00900266.x

48. Demiris G, Parker D, Washington K, Thomas L, Fruehling M, Haggarty-Ro- 
bbins et al. A problem solving intervention for hospice caregivers: A pilot study. J Palliat Med 2010;13:1005-11. Doi:10.1089jpm.2010.0022

49. Meyers FJ, Carducci M, Loscalzo MJ, Linder J, Greasby T, Beckett L. Effects a problem- solving intervention (COPE) on quality of life for patients with advanced cancer on clinical trials and their caregivers. Simultaneous care educational intervention (SCEI): linking palliation and cli- nical trials. J Palliat Med 2011;14:465-73. Doi: 10.1089/jpm.2010.0416.

50. Slaikeu KA. Intervención en crisis: Manual para la práctica e investigación. México, El Manual Moderno, 1996.

51. Galfin JM, Watkins D, Harlow T. A brief guided self-help intervention for psychological distress in palliative care patients: A randomised controlled trial. Palliat Med 2012 26;26:197-205. Doi: $10.1177 / 0269216311414757$ 